\title{
Complications in Patients with Acute Myocardial Infarction Supported with Extracorporeal Membrane Oxygenation
}

\author{
Saraschandra Vallabhajosyula *(D, Malcolm R. Bell, Gurpreet S. Sandhu, Allan S. Jaffe, \\ David R. Holmes Jr. $D$ and Gregory W. Barsness \\ Department of Cardiovascular Medicine, Mayo Clinic, Rochester, MN 55905, USA; \\ Bell.Malcolm@mayo.edu (M.R.B.); Sandhu.Gurpreet@mayo.edu (G.S.S.); Jaffe.Allan@mayo.edu (A.S.J.); \\ Holmes.David@mayo.edu (D.R.H.J.); Barsness.Gregory@mayo.edu (G.W.B.) \\ * Correspondence: Vallabhajosyula.Saraschandra@mayo.edu
}

Received: 19 February 2020; Accepted: 17 March 2020; Published: 19 March 2020

\begin{abstract}
Background: There are limited data on complications in acute myocardial infarction (AMI) admissions receiving extracorporeal membrane oxygenation (ECMO). Methods: Adult ( $>18$ years) admissions with AMI receiving ECMO support were identified from the National Inpatient Sample database between 2000 and 2016. Complications were classified as vascular, lower limb amputation, hematologic, and neurologic. Outcomes of interest included temporal trends, in-hospital mortality, hospitalization costs, and length of stay. Results: In this 17-year period, in 10 million AMI admissions, ECMO support was used in 4608 admissions $(<0.01 \%)$ - mean age $59.5 \pm 11.0$ years, $75.7 \%$ men, $58.9 \%$ white race. Median time to ECMO placement was 1 (interquartile range [IQR] 0-3) day. Complications were noted in 2571 (55.8\%) admissions-vascular 6.1\%, lower limb amputations $1.1 \%$, hematologic $49.3 \%$, and neurologic $9.9 \%$. There was a steady increase in overall complications during the study period ( $21.1 \%$ in 2000 vs. $70.5 \%$ in 2016). The cohort with complications, compared to those without complications, had comparable adjusted in-hospital mortality ( $60.7 \%$ vs. $54.0 \%$; adjusted odds ratio 0.89 [95\% confidence interval 0.77-1.02]; $p=0.10$ ) but longer median hospital stay (12 [IQR 5-24] vs. 7 [IQR 3-21] days), higher median hospitalization costs ( $\$ 458,954$ [IQR 260,522-737,871] vs. 302,255 [IQR 173,033-623,660]), fewer discharges to home (14.7\% vs. 17.9\%), and higher discharges to skilled nursing facilities $(44.1 \%$ vs. $33.9 \%$ ) (all $p<0.001$ ). Conclusions: Over half of all AMI admissions receiving ECMO support develop one or more severe complications. Complications were associated with higher resource utilization during and after the index hospitalization.
\end{abstract}

Keywords: acute myocardial infarction; extracorporeal membrane oxygenation; complications; vascular; hemorrhage

\section{Introduction}

Extracorporeal membrane oxygenation (ECMO) is increasingly used in acute cardiovascular care for the management of acute myocardial infarction (AMI) complicated by cardiac arrest, cardiogenic shock and complications from cardiac interventional procedures [1-10]. ECMO provides cardiac output support of nearly 3-5 L, biventricular support and respiratory support, thereby assisting with critical cardiorespiratory support in extreme situations [11]. Despite limited data and the absence of randomized controlled trials, ECMO has been widely accepted in clinical practice [2-8]. Despite the stated benefits from ECMO therapy, these acutely ill patients receiving ECMO support for AMI continue to experience high in-hospital mortality [7]. As compared to the intra-aortic balloon pump (IABP), the newer mechanical circulatory devices, such as the percutaneous left ventricular assist device (pLVAD) 
and ECMO, are associated with higher rates of complications [1]. There are limited contemporary data on complications in AMI patients receiving ECMO support [1]. As compared to other populations receiving ECMO support such as acute decompensated heart failure and post-cardiotomy cardiogenic shock, AMI patients are unique in the urgency related to insertion, often receive peripheral cannulation, may benefit from left ventricular unloading, may have concomitant cardiac arrest, and constitute $>80 \%$ of cardiogenic shock etiology. Therefore, it is important to understand the complications and outcomes associated with ECMO in AMI. Using a 17-year national database, we sought to systematically evaluate a contemporary United States population.

\section{Material and Methods}

The National (Nationwide) Inpatient Sample (NIS) is the largest all-payer database of hospital inpatient stays in the United States. NIS contains discharge data from a $20 \%$ stratified sample of community hospitals and is a part of the Healthcare Cost and Utilization Project (HCUP), sponsored by the Agency for Healthcare Research and Quality [12]. Information regarding each discharge includes patient demographics, primary payer, hospital characteristics, principal diagnosis, up to 24 secondary diagnoses, and procedural diagnoses. These data are available to other authors via the HCUP-NIS database with the Agency for Healthcare Research and Quality.

During the period between 1 January 2000 through 31 December 2016, a retrospective cohort of admissions from the HCUP-NIS with a primary diagnosis of AMI (International Classification of Disease-9 Clinical Modification [ICD-9CM] 410.x; International Classification of Disease-10 Clinical Modification [ICD-10CM] I21.x-22.x) receiving ECMO support (ICD-9CM 39.65; ICD-10CM 5A15223) were identified consistent with prior literature [7]. Deyo's modification of Charlson Comorbidity Index was used to identify co-morbid diseases and prior methodology was used to identify cardiac and non-cardiac procedures $[2,3,7,8,11,13-23]$. We identified relevant complications and categorized them as (a) vascular complications-arterial injury, acquired arterio-venous fistula, and vascular complications requiring surgery; (b) lower limb amputation; (c) hematologic-post-operative hemorrhage, hemolytic anemia, thrombocytopenia, and blood transfusion; and (d) neurologic-ischemic or hemorrhagic stroke (Supplementary Table S1). The primary outcome was the temporal trend in complications in and secondary outcomes included in-hospital mortality, hospitalization costs and length of stay in AMI admissions supported with ECMO.

\section{Statistical Analysis}

As recommended by HCUP-NIS, survey procedures using discharge weights provided with HCUP-NIS database were used to generate national estimates. Using the trend weights provided by the HCUP-NIS, samples from 2000-2011 were re-weighted to adjust for the 2012 HCUP-NIS re-design. Chi-square and $t$-tests were used to compare categorical and continuous variables respectively. The inherent restrictions of the HCUP-NIS database related to research design, data interpretation, and data analysis were reviewed and addressed. Pertinent considerations include not assessing individual hospital-level volumes (due to changes to sampling design detailed above), treating each entry as an 'admission' as opposed to individual patients, restricting the study details to inpatient factors since the HCUP-NIS does not include outpatient data, and limiting administrative codes to those previously validated and used for similar studies. Univariable analysis for trends, predictors and outcomes was performed and were represented as odds ratio with $95 \%$ confidence interval. Multivariable logistic regression analysis incorporates age, sex, race, primary payer, comorbidity, hospital characteristics, acute organ failure, cardiogenic shock, cardiac arrest, type of AMI, cardiac, and non-cardiac procedures. For the multivariable modeling, regression analysis with purposeful selection of statistically (liberal threshold of $p<0.20$ in univariate analysis) and clinically relevant variables was conducted. Two-tailed $p<0.05$ was considered statistically significant. All statistical analyses were performed using SPSS v25.0 (IBM Corp, Armonk, NY, USA). 


\section{Results}

During this 17-year period, there were over 10 million AMI admissions, of which $4608(<0.01 \%)$ received ECMO support. ST-segment-elevation AMI, cardiogenic shock and cardiac arrest were noted in $66.4 \%, 80.9 \%$ and $45.6 \%$, respectively. Mean age was $59.5 \pm 11.0$ years, $3490(75.7 \%)$ were men, 2716 (58.9\%) were of white race, and mean Charlson Comorbidity Index was $4.1 \pm 2.0$. Of these, over $85 \%$ were admitted to large teaching hospitals. Acute respiratory, renal, hepatic, hematologic, and neurologic dysfunction was noted in $63.2 \%, 63.6 \%, 32.3 \%, 37.4 \%$, and $28.1 \%$ respectively. Coronary angiography, percutaneous coronary intervention, and pulmonary artery/right heart catheterization were used in $52.2 \%, 40.5 \%$, and $23.1 \%$, respectively. Concomitant IABP and pLVAD were used in $49.2 \%$ and $13.5 \%$, respectively. Median time to ECMO placement was 1 day (interquartile range [IQR] 0-3).

Complications were noted in 2571 (55.8\%) admissions-vascular complications 6.1\%, lower limb amputations 1.1\%, hematologic complications 49.3\%, and neurologic complications in 9.9\% (Figure 1A). Thrombocytopenia, need for blood transfusion, and post-operative hemorrhage were the most common complications. The 17-year temporal trends showed a steady increase in complications, primarily due to an increase in hematologic complications (Figure 1B). In a multivariable logistic regression analysis, non-white race, non-Medicare insurance, higher comorbidity, admission to an urban hospital, admission to a medium and large-sized hospital, non-cardiac organ failure, and non-cardiac organ support were associated with development of complications (Supplementary Table S2).

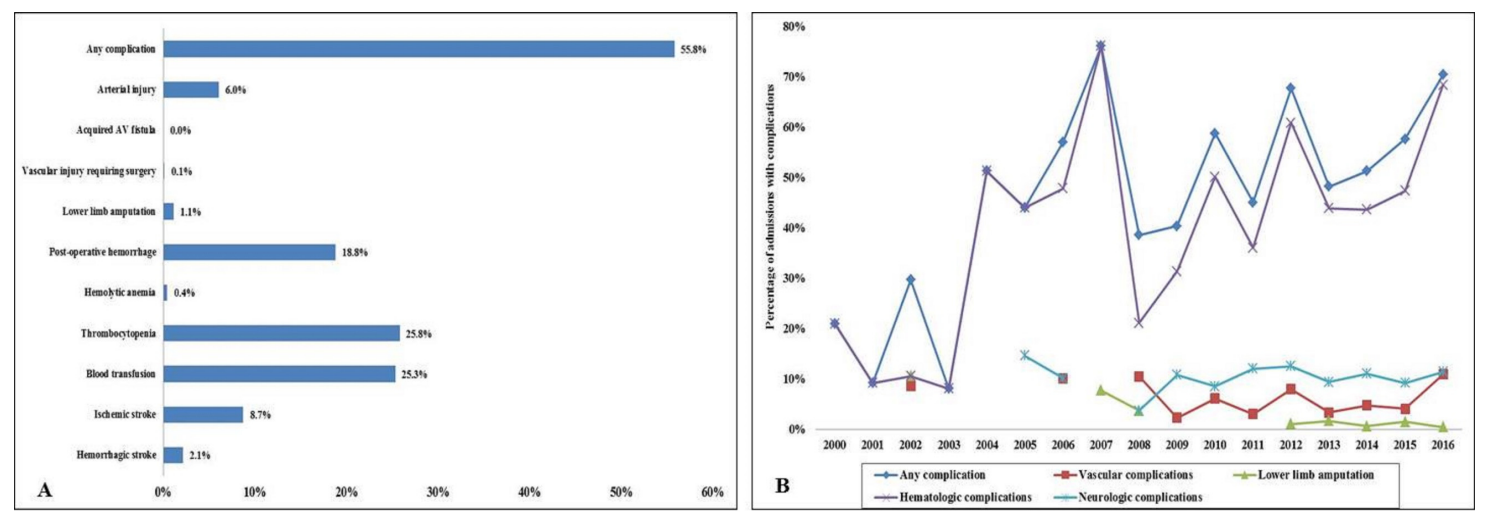

Figure 1. Complications in acute myocardial infarction admissions supported by extracorporeal membrane oxygenation. (A) Relative percentages of in-hospital complications across the various complication categories; (B): 17-year temporal trends of in-hospital complications; $p<0.001$ for trend. AV: arterio-venous.

The cohort with complications, compared to those without, had higher in-hospital mortality (60.7\% vs. 54.0\%; unadjusted odds ratio 1.31 (95\% confidence interval $1.17-1.48) ; p<0.001)$. In a multivariable logistic regression analysis, presence of complications was not independently associated with higher in-hospital mortality in ECMO recipients (odds ratio 0.89 (95\% confidence interval 0.77-1.02); $p=0.10$ ) (Table 1). The cohort with complications had longer median hospital stay (12 (IQR 5-24) vs. 7 (IQR 3-21) days), higher median hospitalization costs $(\$ 458,954$ (IQR 260,522-737,871) vs. 302,255 (IQR 173,033-623,660)), fewer discharges to home (14.7\% vs. 17.9\%), and higher discharges to skilled nursing facilities $(44.1 \%$ vs. $33.9 \%$ ) (all $p<0.001)$. The cohort with complications had longer hospitalization and higher hospitalization costs over the 17-year period (Figure 2A,B). 
Table 1. Predictors of in-hospital mortality.

\begin{tabular}{|c|c|c|c|c|c|}
\hline \multirow{2}{*}{\multicolumn{2}{|c|}{$\begin{array}{l}\text { Overall Cohort } \\
\quad(n=4608)\end{array}$}} & \multirow{2}{*}{ Odds Ratio } & \multicolumn{2}{|c|}{ 95\% Confidence Interval } & \multirow{2}{*}{$p$} \\
\hline & & & Lower Limit & Upper Limit & \\
\hline \multicolumn{2}{|c|}{ Any complication } & 0.89 & 0.77 & 1.02 & 0.10 \\
\hline \multirow{2}{*}{ Age groups (years) } & $\leq 75$ years & \multicolumn{4}{|c|}{ Reference category } \\
\hline & $>75$ years & 1.54 & 1.16 & 2.03 & 0.003 \\
\hline \multirow{2}{*}{ Sex } & Male & \multicolumn{4}{|c|}{ Reference category } \\
\hline & Female & 1.21 & 1.04 & 1.40 & 0.01 \\
\hline \multirow{2}{*}{ Race } & White & \multicolumn{4}{|c|}{ Reference category } \\
\hline & Non-White ${ }^{\text {a }}$ & 1.42 & 1.25 & 1.63 & $<0.001$ \\
\hline \multirow{4}{*}{ Primary payer } & Medicare & \multicolumn{4}{|c|}{ Reference category } \\
\hline & Medicaid & 0.59 & 0.47 & 0.74 & $<0.001$ \\
\hline & Private & 0.49 & 0.41 & 0.57 & $<0.001$ \\
\hline & Others $^{b}$ & 1.02 & 0.80 & 1.29 & 0.89 \\
\hline \multirow{3}{*}{ Charlson Comorbidity Index } & $0-3$ & \multicolumn{4}{|c|}{ Reference category } \\
\hline & $4-6$ & 1.00 & 0.86 & 1.16 & 0.99 \\
\hline & $\geq 7$ & 0.97 & 0.76 & 1.24 & 0.82 \\
\hline \multirow{3}{*}{$\begin{array}{l}\text { Hospital teaching } \\
\text { status and location }\end{array}$} & Rural & \multicolumn{4}{|c|}{ Reference category } \\
\hline & Urban non-teaching & 0.67 & 0.37 & 1.21 & 0.19 \\
\hline & Urban teaching & 1.57 & 0.91 & 2.69 & 0.10 \\
\hline \multirow{3}{*}{ Hospital bed-size } & Small & \multicolumn{4}{|c|}{ Reference category } \\
\hline & Medium & 0.99 & 0.66 & 1.47 & 0.95 \\
\hline & Large & 1.60 & 1.11 & 2.31 & 0.01 \\
\hline \multirow{4}{*}{ Hospital region } & Northeast & \multicolumn{4}{|c|}{ Reference category } \\
\hline & Midwest & 0.93 & 0.78 & 1.11 & 0.44 \\
\hline & South & 0.77 & 0.66 & 0.91 & 0.002 \\
\hline & West & 0.98 & 0.79 & 1.23 & 0.89 \\
\hline \multirow{2}{*}{ AMI type } & STEMI & \multicolumn{4}{|c|}{ Reference category } \\
\hline & NSTEMI & 1.00 & 0.86 & 1.15 & 0.96 \\
\hline \multirow{3}{*}{ Acute organ dysfunction } & Respiratory & 1.18 & 1.02 & 1.35 & 0.02 \\
\hline & Renal & 1.63 & 1.41 & 1.88 & $<0.001$ \\
\hline & Hepatic & 1.42 & 1.23 & 1.65 & $<0.001$ \\
\hline \multicolumn{2}{|c|}{ Cardiogenic shock } & 0.86 & 0.72 & 1.01 & 0.07 \\
\hline \multicolumn{2}{|c|}{ Cardiac arrest } & 1.01 & 0.89 & 1.15 & 0.85 \\
\hline \multicolumn{2}{|c|}{ Coronary angiography } & 1.36 & 1.17 & 1.57 & $<0.001$ \\
\hline Percutaneous coronar & intervention & 0.66 & 0.57 & 0.76 & $<0.001$ \\
\hline Pulmonary artery ca & heterization & 0.66 & 0.54 & 0.81 & $<0.001$ \\
\hline Second mechanical circu & tory support use & 0.97 & 0.85 & 1.11 & 0.66 \\
\hline Invasive mechanica & ventilation & 1.21 & 1.06 & 1.39 & 0.005 \\
\hline Hemodialys & use & 1.81 & 1.39 & 2.35 & $<0.001$ \\
\hline
\end{tabular}

Legend: ${ }^{a}$ Black, Hispanic, Asian, Native American, Others; ${ }^{b}$ Uninsured, No Charge, Others. Abbreviations: AMI: acute myocardial infarction; NSTEMI: non-ST-segment elevation myocardial infarction; STEMI: ST-segment elevation myocardial infarction. 


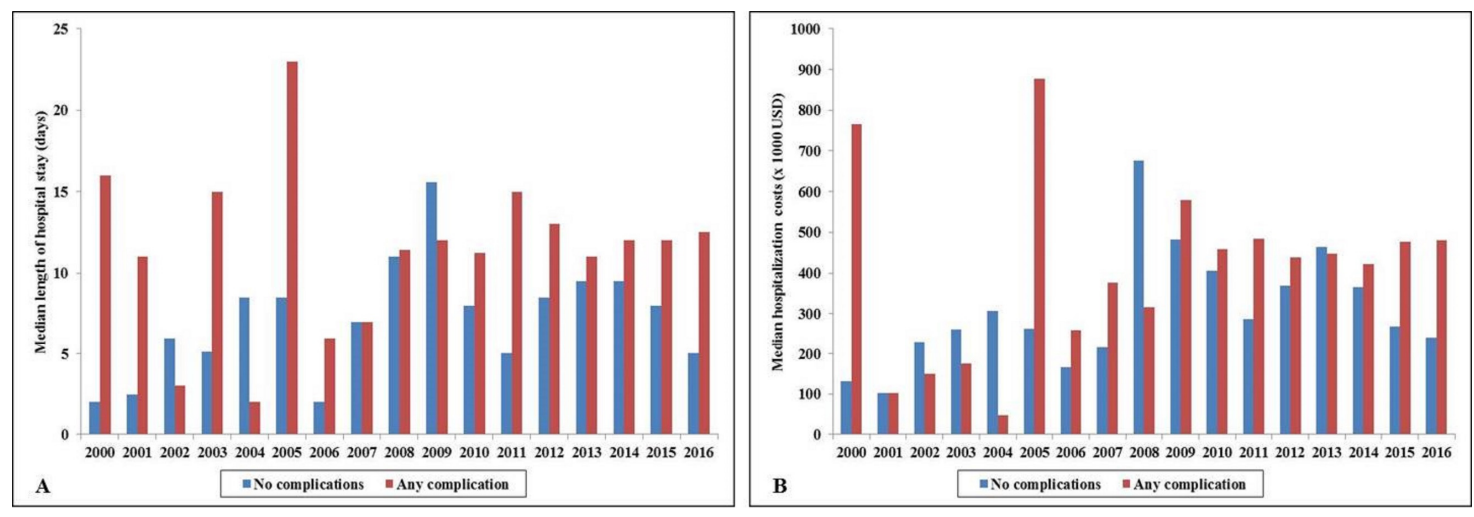

Figure 2. In-hospital resource utilization in acute myocardial infarction admissions supported by extracorporeal membrane oxygenation with and without complications. (A) Seventeen-year temporal trends of median length of stay in admissions with and without complications; $p<0.001$ for trend; (B): 17-year temporal trends of median hospitalization costs in admissions with and without complications; $p<0.001$ for trend. USD: United States Dollars.

\section{Discussion}

In the largest study looking at complications from ECMO use in AMI, nearly $56 \%$ of admissions had $\geq 1$ complication. Hematologic complications, specifically thrombocytopenia, which are needed for blood transfusion and post-operative hemorrhage, were the most commonly noted complications. During this 17-year period, there was a steady increase in complications in AMI admissions receiving ECMO support. The presence of complications was associated with greater resource utilization during and after hospitalization, but had comparable in-hospital mortality to ECMO recipients without complications.

In patients with AMI, ECMO is often used to support tenuous hemodynamics during cardiac arrest, cardiopulmonary resuscitation, cardiogenic shock, and high risk coronary intervention [1-8]. However, by virtue of its unique configuration, ECMO is associated with higher left ventricular afterload, greater vascular complications due to large bore access, limb ischemia due to lack of antegrade flow, greater clotting, and thrombocytopenia due to a complex circuit and higher risk of strokes due to variation in anticoagulation [1]. There are limited data on the complications in AMI patients supported with ECMO [1]. In this study, we report comparable rates of hemorrhage, blood transfusion, arterial injury and limb ischemia, but slightly higher rates of thrombocytopenia compared to prevalent literature [1]. Patients with AMI are unique in that they frequently receive urgent peripheral cannulation, and therefore, carry a higher risk of left ventricular stasis and thrombosis. We have previously reported on the concomitant use of IABP and pLVAD, but are unable to assess if these were placed for left ventricular unloading due to the limitations of an administrative database [4,5,7]. The optimal method of left ventricular unloading in this population remains to be defined [4,5]. In light of the high costs of ECMO support, further careful study of complication rates, more standardized reporting and a detailed risk-benefit discussion are needed to optimize patient outcomes. Lastly, the lower rates of angiography and percutaneous coronary intervention in this study are consistent with prior real-world literature that reflects reluctance to perform angiography in higher risk cohorts despite robust guideline recommendations [7].

This study has several limitations that are inherent to the analysis of a large administrative database. The HCUP-NIS attempts to mitigate potential errors by using internal and external quality control measures. The HCUP-NIS database does not provide further granular information on timing of a procedure beyond hospital day, and therefore, the exact sequence of events (i.e., angiography, coronary intervention, ECMO insertion, and timing of complications) cannot be reliably discerned if all happen within the same hospital day. Information on coronary anatomy, successful revascularization, vasoactive medication use and dosing, left ventricular function, peak serum lactate, and hemodynamic 
variables known to influence outcomes in this population, were unavailable in the HCUP-NIS database. Additionally, because of the non-randomized nature of this study, it is challenging to fully understand the baseline differences in the groups and determine how this has impacted on outcomes. It is conceivable that these patients may have had uniformly high in-hospital mortality in the absence of ECMO support, however, further data are needed from randomized trials. Although in patients with AMI with or without cardiogenic shock, venoarterial ECMO is the most commonly used configuration of ECMO support, it is possible that the ICD-9CM used in this study may refer to the veno-venous ECMO, used for respiratory support (the ICD-10CM codes distinguish these various configurations). Because of the limitations of the administrative coding, this database is unable to distinguish venoarterial and veno-venous configurations. Despite these limitations, this study addresses an important knowledge gap highlighting the national trends and outcomes of in-hospital complications in AMI admissions receiving ECMO support.

In summary, complications in AMI admissions receiving ECMO support have resulted in greater resource utilization during and after the index hospitalization, despite comparable in-hospital mortality. Given the high costs associated with ECMO support, optimal patient and device selection is key in this critically ill population.

Supplementary Materials: The following are available online at http://www.mdpi.com/2077-0383/9/3/839/s1, Table S1: Administrative codes, Table S2: Predictors of complications in AMI admissions receiving ECMO.

Author Contributions: Study design, literature review, data analysis, statistical analysis: S.V., G.W.B. Data management, data analysis, drafting manuscript: S.V., G.W.B. Access to data: S.V., M.R.B., G.S.S., A.S.J., D.R.H., G.W.B. Manuscript revision, intellectual revisions, mentorship: M.R.B., G.S.S., A.S.J., D.R.H., G.W.B. Final approval: S.V., M.R.B., G.S.S., A.S.J., D.R.H., G.W.B. All authors have read and agreed to the published version of the manuscript.

Funding: S.V. is supported by the Clinical and Translational Science Award (CTSA) Grant Number UL1 TR000135 from the National Center for Advancing Translational Sciences (NCATS), a component of the National Institutes of Health (NIH). Its contents are solely the responsibility of the authors and do not necessarily represent the official view of NIH.

Conflicts of Interest: A.S.J. has been a consultant for Beckman, Abbott, Siemens, Roche, ET Healthcare, Sphingotoec, Quidel, Brava, Blade, and Novartis. All other authors have reported that they have no relationships relevant to the contents of this paper to disclose.

$\begin{array}{ll}\text { Abbreviations } \\ \text { AMI } & \text { acute myocardial infarction } \\ \text { ECMO } & \text { extracorporeal membrane oxygenation } \\ \text { HCUP } & \text { Healthcare Cost and Utilization Project } \\ \text { IABP } & \text { intra-aortic balloon pump } \\ \text { ICD-9CM } & \text { International Classification of Diseases-9 Clinical Modification } \\ \text { ICD-10CM } & \text { International Classification of Diseases-10 Clinical Modification } \\ \text { IQR } & \text { interquartile range } \\ \text { NIS } & \text { National/Nationwide Inpatient Sample } \\ \text { pLVAD } & \text { percutaneous left ventricular assist device }\end{array}$

\section{References}

1. Subramaniam, A.V.; Barsness, G.W.; Vallabhajosyula, S.; Vallabhajosyula, S. Complications of temporary percutaneous mechanical circulatory support for cardiogenic shock: an appraisal of contemporary literature. Cardiol. Ther. 2019, 8, 211-228.

2. Vallabhajosyula, S.; Arora, S.; Lahewala, S.; Kumar, V.; Shantha GP, S.; Jentzer, J.C.; Stulak, J.M.; Gersh, B.J.; Gulati, R.; Rihal, C.S.; et al. Temporary mechanical circulatory support for refractory cardiogenic shock before left ventricular assist device surgery. J. Am. Heart Assoc. 2018, 7, e010193. [CrossRef]

3. Vallabhajosyula, S.; Arora, S.; Sakhuja, A.; Lahewala, S.; Kumar, V.; Shantha, G.P.S.; Egbe, A.C.; Stulak, J.M.; Gersh, B.J.; Gulati, R.; et al. Trends, predictors, and outcomes of temporary mechanical circulatory support for postcardiac surgery cardiogenic shock. Am. J. Cardiol. 2019, 123, 489-497. 
4. Vallabhajosyula, S.; O’Horo, J.C.; Antharam, P.; Ananthaneni, S.; Vallabhajosyula, S.; Stulak, J.M.; Dunlay, S.M.; Holmes, D.R.; Barsness, G.W. Venoarterial extracorporeal membrane oxygenation with concomitant Impella versus venoarterial extracorporeal membrane oxygenation for cardiogenic shock. ASAIO J. 2019. [CrossRef] [PubMed]

5. Vallabhajosyula, S.; O’Horo, J.C.; Antharam, P.; Ananthaneni, S.; Vallabhajosyula, S.; Stulak, J.M.; Eleid, M.F.; Dunlay, S.M.; Gersh, B.J.; Rihal, C.S.; et al. Concomitant intra-aortic balloon pump use in cardiogenic shock requiring veno-arterial extracorporeal membrane oxygenation. Circ. Cardiovasc Interv. 2018, 11, e006930. [CrossRef] [PubMed]

6. Vallabhajosyula, S.; Patlolla, S.H.; Sandhyavenu, H.; Vallabhajosyula, S.; Barsness, G.W.; Dunlay, S.M.; Greason, K.L.; Holmes, D.R.; Eleid, M.F. Periprocedural cardiopulmonary bypass or venoarterial extracorporeal membrane oxygenation during transcatheter aortic valve replacement: a systematic review. J. Am. Heart Assoc. 2018, 7, e009608. [CrossRef] [PubMed]

7. Vallabhajosyula, S.; Prasad, A.; Bell, M.R.; Sandhu, G.S.; Eleid, M.F.; Dunlay, S.M.; Schears, G.J.; Stulak, J.M.; Singh, M.; Gersh, B.J.; et al. Extracorporeal membrane oxygenation use in acute myocardial infarction in the United States, 2000 to 2014. Circ. Heart Fail. 2019, 12, e005929. [CrossRef]

8. Vallabhajosyula, S.; Prasad, A.; Sandhu, G.S.; Gulati, R.; Eleid, M.F.; Best, P.J.M.; Gersh, B.J.; Singh, M.; Lerman, A.; Holmes, D.R.; et al. Mechanical Circulatory support-assisted early percutaneous coronary intervention in acute myocardial infarction with cardiogenic shock: 10-year national temporal trends, predictors and outcomes. EuroIntervention. 2019. [CrossRef]

9. Vallabhajosyula, S.; Barsness, G.W.; Vallabhajosyula, S. Multidisciplinary teams for cardiogenic shock. Aging 2019, 11, 4774-4776. [CrossRef]

10. Vallabhajosyula, S.; Vallabhajosyula, S.; Vaidya, V.R.; Patlolla, S.H.; Desai, V.; Mulpuru, S.K.; Noseworthy, P.A.; Suraj, K.; Egbe, A.C.; Gersh, B.J.; et al. Venoarterial extracorporeal membrane oxygenation support for ventricular tachycardia ablation: a systematic review. ASAIO J. 2020. [CrossRef]

11. Vallabhajosyula, S.; Kashani, K.; Dunlay, S.M.; Vallabhajosyula, S.; Vallabhajosyula, S.; Sundrargiri, P.R.; Gersh, B.J.; Jaffe, A.S.; Barsness, G.W. Acute respiratory failure and mechanical ventilation in cardiogenic shock complicating acute myocardial infarction in the USA, 2000-2014. Ann. Intensive Care. 2019, 9, 96. [CrossRef] [PubMed]

12. Introduction to the HCUP Nationwide Inpatient Sample 2009. Available online: http://www.hcup-us.ahrq. gov/db/nation/nis/NIS_2009_INTRODUCTION.pdf (accessed on 18 January 2015).

13. Vallabhajosyula, S.; Dunlay, S.M.; Barsness, G.W.; Rihal, C.S.; Holmes, D.R., Jr.; Prasad, A. Hospital-level disparities in the outcomes of acute myocardial infarction with cardiogenic shock. Am. J. Cardiol. 2019, 124, 491-498. [CrossRef] [PubMed]

14. Vallabhajosyula, S.; Dunlay, S.M.; Barsness, G.W.; Vallabhajosyula, S.; Vallabhajosyula, S.; Sundrargiri, P.R.; Gersh, B.J.; Jaffe, A.S.; Kashani, K. Temporal trends, predictors, and outcomes of acute kidney injury and hemodialysis use in acute myocardial infarction-related cardiogenic shock. PLOS ONE 2019, 14, e0222894. [CrossRef] [PubMed]

15. Vallabhajosyula, S.; Dunlay, S.M.; Kashani, K.; Vallabhajosyula, S.; Vallabhajosyula, S.; Sundrargiri, P.R.; Jaffe, A.S.; Barsness, G.W. Temporal trends and outcomes of prolonged invasive mechanical ventilation and tracheostomy use in acute myocardial infarction with cardiogenic shock in the United States. Int J. Cardiol. 2019, 285, 6-10. [CrossRef] [PubMed]

16. Vallabhajosyula, S.; Dunlay, S.M.; Murphree, D.H.; Barsness, G.W.; Sandhu, G.S.; Lerman, A.; Prasad, A. Cardiogenic shock in takotsubo cardiomyopathy versus acute myocardial infarction: an 8-year national perspective on clinical characteristics, management, and outcomes. JACC Heart Fail. 2019, 7, 469-476. [CrossRef] [PubMed]

17. Vallabhajosyula, S.; Dunlay, S.M.; Prasad, A.; Kashani, K.; Sakhuja, A.; Gersh, B.J.; Jaffe, A.S.; Holmes, D.R.; Barsness, G.W. Acute noncardiac organ failure in acute myocardial infarction with cardiogenic shock. J. Am. Coll Cardiol. 2019, 73, 1781-1791. [CrossRef]

18. Vallabhajosyula, S.; El Hajj, S.C.; Bell, M.R.; Prasad, A.; Lerman, A.; Rihal, C.S.; Holmes, D.R.; Barsness, G.W. Intravascular ultrasound, optical coherence tomography, and fractional flow reserve use in acute myocardial infarction. Catheter Cardiovasc Interv. 2019. [CrossRef] 
19. Vallabhajosyula, S.; Patlolla, S.H.; Dunlay, S.M.; Prasad, A.; Bell, M.R.; Jaffe, A.; Gersh, B.J.; Rihal, C.S.; Holmes, D.R.; Barsness, G.W. Regional variation in the management and outcomes of acute myocardial infarction with cardiogenic shock in the United States. Circ. Heart Fail. 2020, 13, e006661. [CrossRef]

20. Vallabhajosyula, S.; Prasad, A.; Dunlay, S.M.; Murphee, D.H.; Ingram, C.; Mueller, P.S.; Gersh, B.J.; Holmes, D.R.; Barsness, G.W. Utilization of palliative care for cardiogenic shock complicating acute myocardial infarction: A 15-year national perspective on trends, disparities, predictors, and outcomes. J. Am. Heart Assoc. 2019, 8, e011954. [CrossRef]

21. Vallabhajosyula, S.; Prasad, A.; Gulati, R.; Barsness, G.W. Contemporary prevalence, trends, and outcomes of coronary chronic total occlusions in acute myocardial infarction with cardiogenic shock. Int J. Cardiol Heart Vasc. 2019, 24, 100414. [CrossRef]

22. Vallabhajosyula, S.; Vallabhajosyula, S.; Bell, M.R.; Prasad, A.; Singh, M.; White, R.D.; Jaffe, A.S.; Holmes, D.R.; Jentzer, J.C. Early vs. delayed in-hospital cardiac arrest complicating ST-elevation myocardial infarction receiving primary percutaneous coronary intervention. Resuscitation 2019. [CrossRef] [PubMed]

23. Vallabhajosyula, S.; Ya'Qoub, L.; Dunlay, S.M.; Vallabhajosyula, S.; Vallabhajosyula, S.; Sundaragiri, P.R.; Jaffe, A.S.; Gersh, B.J.; Kashani, K. Sex disparities in acute kidney injury complicating acute myocardial infarction with cardiogenic shock. ESC Heart Fail. 2019, 6, 874-877. [CrossRef] [PubMed]

(C) 2020 by the authors. Licensee MDPI, Basel, Switzerland. This article is an open access article distributed under the terms and conditions of the Creative Commons Attribution (CC BY) license (http://creativecommons.org/licenses/by/4.0/). 